\title{
Normal Smoothings for Smooth Cube Manifolds
}

\author{
Pedro Ontaneda*
}

\begin{abstract}
A smooth cube manifold $M^{n}$ is a smooth $n$-manifold $M$ together with a smooth cubulation on $M$. (A smooth cubulation is similar to a smooth triangulation, but with cubes instead of simplices). The cube structure provides, for each open $k$-subcube $\dot{\sigma}^{k}$, rays that are normal to $\dot{\sigma}$. Using these rays we can construct normal charts of the form $\mathbb{D}^{n-k} \times \dot{\sigma} \rightarrow M$, where we are identifying $\mathbb{D}^{n-k}$ with the cone over the link of $\dot{\sigma}$ (these identifications are arbitrary and the identification between $\partial \mathbb{D}^{n-k}$ and the link of $\dot{\sigma}$ is called a link smoothing). These normal charts respect the product structure of $\mathbb{D}^{n-k} \times \dot{\sigma}$ and the radial structure of $\mathbb{D}^{n-k}$. A complete set of normal charts gives a (topological) normal atlas on $M$. If this atlas is smooth it is called a normal smooth atlas on $M$ and induces a normal smooth structure on $M$ (normal with respect to the cube structure). In this paper we prove that every smooth cube manifold has a normal smooth structure, which is diffeomorphic to the original one. This result also holds for smooth all-right-spherical manifolds.
\end{abstract}

\section{Section 0. Introduction.}

As mentioned in the Abstract, in this paper we address the following quite natural question. Given a a cube complex (very popular objects these days) one can construct "normal charts" in an obvious natural way (charts respect radial directions). Question: given a smooth cube complex (smooth manifold + compatible cube structure), does there exist a normal smooth structure? (i.e is there a smooth atlas formed of normal charts?) In this paper we give an affirmative answer to this question.

The results in this paper are key ingredients to smooth the metric of a strictly hyperbolized manifold (see [12] and [11]). This smoothing implies the existence of a large class of negatively curved Riemannian manifolds, showing that, in some sense, Riemanian negative curvature abounds in nature.

Here is a brief explanation how normal smooth structures on cube manifolds are used in [12]. To any manifold $M$ with a smooth cubulation $K$ one can associate a smooth piecewise hyperbolic manifold $K_{X}$ produced via the strict hyperbolization procedure of Charney and Davis (based on Gromov's hyperbolization process). Using a smooth normal atlas $\mathcal{A}$ on $M$ with respect to $K$, we can contruct a similar "normal" smooth atlas $\mathcal{A}_{K_{X}}$ on the strictly hyperbolized manifold $K_{X}$ (this is done in detail in [11], and also briefly explained in [12]). Because of the form of normal charts (they preserve some of the "cube structure"), it is possible to use the atlas $\mathcal{A}_{K_{X}}$ to smooth the natural piecewise hyperobolic metric on $K_{X}$ via local warped product constructions.

\footnotetext{
${ }^{*}$ The author was partially supported by a NSF grant.
} 
We next give a more detailed description of normal structures and state the Main Theorem.

For the basic definitions and results about cube and spherical complexes see for instance [1]. Recall that a spherical complex is an all-right-spherical complex if all of its edge lengths are equal to $\pi / 2$. Given a (cube or all-right-spherical) complex $K$ we use the same notation $K$ for the complex itself (the collection of all closed cubes or simplices) and its realization (the union of all cubes or simplices). For $\sigma \in K$ we denote its interior by $\dot{\sigma}$. In this paper we assume that all cube or all-right-spherical complexes satisfy the following condition: any two closed cubes or simplices intersect on at most one (possibly empty) common subcube or subsimplex.

Let $M^{n}$ be a smooth manifold of dimension $n$. Recall that a cubulation of $M$ is given by $(K, f)$, where $K$ is a cube complex and $f: K \rightarrow M$ is a homeomorphism. The cubulation is smooth if $f$ a non-degenerate $P D$ homeomorphism [10, that is, for all $\sigma \in K$ we have $\left.f\right|_{\sigma}$ is a smooth embedding. Sometimes we will write $K$ instead of $(K, f)$. The smooth manifold $M$ together with a smooth cubulation is a smooth cube manifold. A smooth all-right-spherical manifold is defined analogously.

In this paper we consider $\operatorname{Link}\left(\sigma^{j}, K\right)$, the (geometric) link of an open $j$-cube or $j$-all-right simplex $\sigma^{j}$, as the union of the end points of straight segments of small length $\epsilon>0$ emanating perpendicularly (to $\dot{\sigma}^{j}$ ) from some fixed point $x \in \dot{\sigma}^{j}$. We say that the link is based at $x$. And the (open) star $\operatorname{Star}(\sigma, K)$ as the union of such half-open $\epsilon$-segments based at x. We can identify the star with the (open) cone of the link $\mathrm{C} \operatorname{Link}(\sigma, K)$ (or $\epsilon$-cone) defined as $\mathrm{C} \operatorname{Link}(\sigma, K)=$ $\operatorname{Link}(\sigma, K) \times[0, \epsilon) / \operatorname{Link}(\sigma, K) \times\{0\}$. We shall denote the cone point by $o$ or, more specifically, by $o_{\mathrm{C} \operatorname{Link}(\sigma, K)}$. Thus a point $x$ in $\mathrm{C} \operatorname{Link}(\sigma, K)$, different from the cone point $o$, can be written as $x=t u, t \in(0, \epsilon), u \in \operatorname{Link}(\sigma, K)$. For $s>0$ we get the cone homothety $x \mapsto s x=(s t) u$ (partially defined if $s>1$ ). If we want to make explicit the dependence of the link or the cone on $\epsilon$ we shall write $\operatorname{Link}_{\epsilon}(\sigma, K)$ or $\mathrm{C}_{\epsilon} \operatorname{Link}(\sigma, K)$ respectively. Also, we will always take $\epsilon<1 / 2(<\pi / 4$ in the spherical case) and it can be verified that all results in this paper (unless otherwise stated) are independent of the choice of the $\epsilon$ 's. As usual we shall identify the $\epsilon$-normal neighborhood of $\dot{\sigma}$ in $K$ with $\mathrm{C}_{\epsilon} \operatorname{Link}(\sigma, K) \times \dot{\sigma}$ (or just $\left.\mathrm{C} \operatorname{Link}(\sigma, K) \times \dot{\sigma}\right)$. Hence a cone homothety induces a neighborhood homothety obtained by crossing it with the identity $1_{\dot{\sigma}}$.

Recall that the link $\operatorname{Link}\left(\sigma^{i}, K\right), \sigma^{i} \in K$, has a natural all-right piecewise spherical structure, which induces a simplicial structure and thus a $P L$ structure on $\operatorname{Link}\left(\sigma^{i}, K\right)$.

From now on we assume that $K$ is a $P L$ manifold, and $f: K \rightarrow L$ a homoemorphism. (For instance, $K$ is a smooth cubulation, or triangulation, of $M$; in this case the $P L$ structure on $M$ induced by $K$ is Whitehead compatible with $M$, hence $K$ is a $P L$ manifold, see [7], p.10.) Therefore the link Link $\left(\sigma^{i}, K\right)$ is $P L$ homeomorphic to $\mathbb{S}^{n-i-1}$, for every $\sigma^{i} \in K$. A link smoothing for $\dot{\sigma}^{i}\left(\right.$ or $\left.\sigma^{i}\right)$ is just a homeomorphism $h_{\sigma^{i}}: \mathbb{S}^{n-i-1} \rightarrow \operatorname{Link}\left(\sigma^{i}, K\right)$. The cone of $h_{\sigma^{i}}$ is the map

$$
\mathrm{C} h_{\sigma^{i}}: \mathbb{D}^{n-i} \longrightarrow \mathrm{C} \operatorname{Link}\left(\sigma^{i}, K\right)
$$

given by $t x=[x, t] \mapsto t h_{q^{i}}(x)=\left[h_{q^{i}}(x), t\right]$, where we are canonically identifying the $\epsilon$-cone of $\mathbb{S}^{n-i-1}$ with the open disc $\mathbb{D}^{n-i}$. We remark that we are not assuming $h_{\sigma^{i}}$ to be $P L$.

A link smoothing $h_{\sigma^{i}}$ induces the following smoothing of the normal neighborhood of $\dot{\sigma}^{i}$ :

$$
h_{\sigma^{i}}^{\bullet}=f \circ\left(\mathrm{C} h_{\sigma^{i}} \times 1_{\dot{\sigma}^{i}}\right): \mathbb{D}^{n-i} \times \dot{\sigma}^{i} \longrightarrow M
$$


The pair $\left(h_{\sigma^{i}}^{\bullet}, \mathbb{D}^{n-i} \times \dot{\sigma}^{i}\right)$, or simply $h_{\sigma^{i}}^{\bullet}$, is a normal chart on $M$. Note that the collection $\mathcal{A}=$ $\left\{\left(h_{\sigma^{i}}^{\bullet}, \mathbb{D}^{n-i} \times \dot{\sigma}^{i}\right)\right\}_{\sigma^{i} \in K}$ is a topological atlas for $M$. Sometimes will just write $\mathcal{A}=\left\{h_{\sigma^{i}}^{\bullet}\right\}_{\sigma^{i} \in K}$. The topological atlas $\mathcal{A}$ depends uniquely on the the complex $K$, the map $f$ and the collection of link smoothings $\left\{h_{\sigma}\right\}_{\sigma \in K}$. To express the dependence of the atlas on the set of links smoothings we shall write $\mathcal{A}=\mathcal{A}\left(\left\{h_{\sigma}\right\}_{\sigma \in K}\right)$ (this is different from $\mathcal{A}=\left\{h_{\sigma^{i}}^{\bullet}\right\}_{\sigma^{i} \in K}$, as written above).

The most important feature about these normal atlases is that they preserve the radial and sphere (link) structure given by $K$. These features make normal atlases very powerful tools for geometric constructions.

Note that not every collection of link smoothings induce a smooth atlas, but when the atlas is smooth we call $\mathcal{A}$ a normal smooth atlas on $M$ with respect to $K$ and the corresponding smooth structure $\mathcal{S}^{\prime}$ a normal smooth structure on $M$ with respect to $K$. Notice that in this case the maps $\left.f\right|_{\dot{\sigma}^{i}}: \dot{\sigma}^{i} \rightarrow\left(M, \mathcal{S}^{\prime}\right)$ are smooth embeddings. Also note that $\mathcal{A}$ is smooth if and only if there is a smooth structure $\mathcal{S}^{\prime}$ such that all normal charts $h_{\sigma^{i}}^{\bullet}: \mathbb{D}^{n-i} \times \dot{\sigma}^{i} \longrightarrow\left(M, \mathcal{S}^{\prime}\right)$ are smooth embeddings. Here is our main result.

Main Theorem. Let $M$ be a smooth cube manifold, with smooth structure $\mathcal{S}$. Then $M$ admits a normal smooth structure $\mathcal{S}^{\prime}$ diffeomorphic to $\mathcal{S}$.

Specifically, if $M^{n}$ is a smooth manifold with smooth structure $\mathcal{S}$ and $K$ is a smooth cubulation of $M$, then we can choose link smoothings $h_{\sigma^{i}}$, for all $\sigma^{i} \in K$, such that the atlas $\mathcal{A}=\mathcal{A}\left(\left\{h_{\sigma}\right\}_{\sigma \in K}\right)$ is smooth. The smooth atlas $\mathcal{A}$ is normal with respect to $K$. Moreover the normal smooth structure $\mathcal{S}^{\prime}$ (with respect to $K$ ), induced by $\mathcal{A}$, is diffeomorphic to $\mathcal{S}$.

Addendum to Main Theorem. The statement of the Main Theorem also holds for smooth all-right-spherical complexes.

\section{Remarks.}

1. It can be checked from the proof of the Main Theorem that in fact $\mathcal{S}^{\prime}$ is isotopic to $\mathcal{S}$ for $n \neq 4$. That is, there is a diffeomorphism $\phi:(M, \mathcal{S}) \rightarrow\left(M, \mathcal{S}^{\prime}\right)$ that is (topologically) isotopic to the identity map $1_{M}$. With some care we can probably include the case $n=4$.

2. Note that the image of the chart $h_{\sigma}^{\bullet}$ is the open normal neighborhood $\stackrel{\circ}{N}_{\epsilon}(\dot{\sigma}, K)$ of width $\epsilon$ of $\dot{\sigma}$ in $K$. Even though we are assuming, for simplicity, that $\epsilon<1 / 2(\epsilon<\pi / 4$ in the spherical case) it can be checked from the proof of the Main Theorem that we can actually take $\epsilon=1(\epsilon=\pi / 2)$ for the charts.

3. The proof of the Main Theorem uses an inductive construction of a normal atlas. The idea is to keep adding normal charts. The main ingredient in the construction is Lemma 1.2 which says that the links of cubes are smooth submanifolds. Lemma 1.2 does not work if we replace cubes by (linear) simplices, or even by equilateral simplices: a key ingredient in the proof of Lemma 1.2. is the smoothness of the function in formula (1), but in the equilateral simplicial case the analogous formula has a function which is not smooth. Of course, if we "reparametrize" the rays in the linear case then the proof works, because this is just the all-right-spherical case.

4. Every smooth cubulation induces a smooth triangulation (by subdivision) and every smooth triangulation induces a smooth cubulation (by canonically "cubifying" the standard simplices). Therefore every smooth manifold admits a smooth cubulation, hence by the Main Theorem, every smooth manifold admits a normal smooth structure, relative to some cube structure. 
5. As application of Lemma 1.2 we get a simple proof of the following fact: the vanishing of $\Theta_{n}$ (the group of homotopy $n$-spheres), $n \leq 6, n \neq 4$, implies that every $P L n$-manifold is smoothable, for $n \leq 7$. (See remark before Proposition 1.4.)

6. The Main Theorem is of course not true if we replace "smooth cubulation" by just "cubulation". In particular the Main Theorem is not true if $K$ is not a $P L$ manifold.

7. Let $\mathcal{S}^{\prime}$ be as in the Main Theorem. As mentioned above the maps $\left.f\right|_{\dot{\sigma}^{i}}: \dot{\sigma}^{i} \rightarrow\left(M, \mathcal{S}^{\prime}\right)$ are smooth embeddings (recall $\dot{\sigma}$ is an open simplex), but we can not expect the maps $\left.f\right|_{\sigma^{i}}$ to be embeddings (recall $\sigma$ is a closed simplex). Nor we can expect the map $f: K \rightarrow\left(M, \mathcal{S}^{\prime}\right)$ to be $\mathrm{PD}$. That is, we cannot expect $(K, f)$ to be a smooth cubulation of $\left(M, \mathcal{S}^{\prime}\right)$. This does not even happen in the next simple example. (On the other hand, since $\mathcal{S}$ and $\mathcal{S}^{\prime}$ are diffeomorphic $(K, \psi)$ is a smooth cubulation of $\left(M, \mathcal{S}^{\prime}\right)$ for some other map $\psi$. In fact this map $\psi$ can also be constructed rather explicitly by induction using Lemma A.2.2 in the appendix; see A.2.5. Lemma A.2.2 corrects the lack of differentiability showed in the next example.)

Example. Consider $\mathbb{R}^{2}$ with its canonical cube structure, that is, the vertices of the cubes are points in $\mathbb{R}^{2}$ with integer coordinates. Thus the 2 -cubes are translations of $[0,1]^{2}$. Note that the canonical smooth structure of $\mathbb{R}^{2}$ is normal with respect to $K$ : choose the normal charts $h_{\sigma^{1}}^{\bullet}$ of 1-cubes to be the obvious product charts, and the link smoothings $h_{\sigma^{0}}$ for the vertices to be translations of the inclusion $\mathbb{S}^{1}=\mathbb{S}_{\epsilon}^{1} \rightarrow \mathbb{R}^{2}$. Consider now $\mathbb{R}^{2}$ with a cube structure given by pulling back the canonical one using the map $p_{\ell}: \mathbb{R}^{2} \rightarrow \mathbb{R}^{2}, p_{\ell}(r, \theta)=(r, \ell \theta)$, where we are using polar coordinates, and $\ell \neq \pm 1$ is an integer. Note that $p_{\ell}$ is not differentiable at the origin. Let $K_{\ell}$ be this new cube complex and $f: K_{\ell} \rightarrow \mathbb{R}^{2}$ be the inclusion. Hence the images of the open cubes $f\left(\dot{\sigma}^{\prime}\right), \sigma^{\prime} \in K_{\ell}$ are the components of $p_{\ell}^{-1}(\dot{\sigma}), \sigma \in K$. Notice that the canonical smooth structure of $\mathbb{R}^{2}$ is also normal with respect to $K_{\ell}$ : (1) choose the normal charts of an open cube $\dot{\sigma}$ in $K_{\ell}, \sigma \neq 0$ ( i.e. a cube different from the origin) to be the composition of $p_{\ell}^{-1}$ with the the normal chart of $p_{\ell}(\sigma)$, that is, choose $h_{\sigma}^{\bullet}=p_{\ell}^{-1} \circ h_{p_{\ell}(\sigma)}^{\bullet}$ (this is well defined), (2) for the origin choose the link smoothing $h_{\ell}$ of the link $\operatorname{Link}\left(0, K_{\ell}\right)$ of the origin as the lifting by $p_{\ell}$ of the link smoothing $h$ of $\operatorname{Link}(0, K)$ (which is just the inclusion):

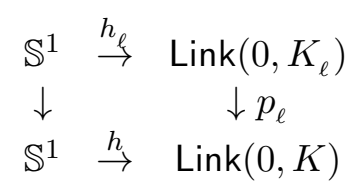

where the left vertical arrow is the map $z \mapsto z^{l}, z \in \mathbb{S}^{1}$. Then for any closed 2-cube $\sigma^{2} \in K_{\ell}$ containing the origin we have that $\left.f\right|_{\sigma^{2}}: \sigma^{2} \rightarrow \mathbb{R}^{2}$ coincides with $\left.p_{\ell}\right|_{\sigma^{2}}$ which is not differentiable at the origin, hence $f$ is not $P D$.

So, even though the normal charts $h_{\sigma^{i}}^{\bullet}$ have potent geometric properties, the example above shows that the $P L$ structure given by $K$ has to be sacrificed. (But we can replace it by an equivalent one).

In section 1 we prove the Main Theorem and in section 2 we generalize our results to the case of manifolds with discrete point singularities. There is one appendix.

We are grateful to the referee for his/her comments and suggestions. 


\section{Section 1. Proof of Main Theorem.}

To simplify our notation we will write the proof for cubical complexes $K$; the proof for the allright-spherical complex case is similar. We will denote an open $i$-cube by $q^{i}$ and the corresponding closed cube by $\bar{q}^{i}$. We write $\operatorname{Link}(q, K)=\operatorname{Link}(\bar{q}, K)$. Also we denote by $K_{j}$ the $j$-skeleton of $K$, i.e. the union of all cubes of dimension $\leq j$. Also write $M_{j}=f\left(K_{j}\right)$. We will prove the Theorem by induction on $k=n-j$. Consider the following statement:

$\mathbf{S}(k)$ There is a smooth structure $\mathcal{S}_{k}$ on $M-M_{j}$ and link smoothings $h_{q^{i}}$ for all $i$-cubes, $i>j$, such that the corresponding normal charts $h_{q^{i}}^{\bullet}: \mathbb{D}^{n-i} \times q^{i} \longrightarrow\left(M-M_{j}, \mathcal{S}_{k}\right)$ are smooth embeddings.

Remark. If $\mathbf{S}(k)$ holds, then $\left\{\left(h_{q^{i}}^{\bullet}, \mathbb{D}^{n-i} \times q^{i}\right)\right\}_{\bar{q}^{i} \in K, i>j}$ is a smooth atlas for $\left(M-M_{j}, \mathcal{S}_{k}\right)$. Moreover, the smooth structure $\left.\mathcal{S}_{k}\right|_{M-M_{j+1}}$ coincides with $\mathcal{S}_{k-1}$, provided the link smoothings of both structures coincide for all the $q^{i}, i>j+1$. Also the maps $\left.f\right|_{q^{i}}: q^{i} \rightarrow\left(M-M_{j}, \mathcal{S}_{k}\right)$ are embeddings, $i>k$.

The following is a key ingredient in the proof of the Main Theorem and is the reason why everything works.

Lemma 1.2. Assume $\mathbf{S}(k)$ holds. Then $f\left(\operatorname{Link}\left(q^{j}, K\right)\right)$ is a smooth submanifold of $\left(M-M_{j}, \mathcal{S}_{k}\right)$, for every $j$-cube $q^{j}$ of $K$.

In general $f\left(\operatorname{Link}\left(q^{j}, K\right)\right)$ may not be diffeomorphic to $\mathbb{S}^{k-1}$, but if it is, we have the following addition to Lemma 1.2.

Addendum to Lemma 1.2. Let $h_{q^{j}}: \mathbb{S}^{k-1} \rightarrow \operatorname{Link}\left(q^{j}, K\right)$ be a link smoothing such that $f \circ h_{q^{j}}$ is a diffeomorphism. Then the corresponding normal chart

$$
\left.h_{q^{j}}^{\bullet}\right|_{\left(\mathbb{D}^{k}-0\right) \times q^{j}}:\left(\mathbb{D}^{k}-0\right) \times q^{j} \rightarrow\left(M-M_{j}, \mathcal{S}_{k}\right)
$$

is a smooth embedding.

Proof of Lemma 1.2. By the remark above it is enough to verify that $f\left(\operatorname{Link}\left(q^{j}, K\right)\right)$ is a smooth submanifold in every chart $h_{q^{i}}^{\bullet}: \mathbb{D}^{n-i} \times q^{i} \longrightarrow\left(M-M_{j}, \mathcal{S}_{k}\right)$, that is, we have to show that $\left(h_{q^{i}}^{\bullet}\right)^{-1}\left(f\left(\operatorname{Link}\left(q^{j}, K\right)\right)\right)$ is a submanifold of $\mathbb{D}^{n-i} \times q^{i}$. We can assume that $\bar{q}^{j}$ is a subcube of $\bar{q}^{i}$. To be specific consider $\operatorname{Link}\left(q^{j}, K\right)=\operatorname{Link}_{\epsilon}\left(q^{j}, K\right), \operatorname{Link}\left(q^{i}, K\right)=\operatorname{Link}_{\delta}\left(q^{i}, K\right)$ and assume that $\operatorname{Link}\left(q^{j}, K\right)$ is based at $x \in q^{j}$. Write $S=\left(h_{q^{i}}^{\bullet}\right)^{-1}\left(f\left(\operatorname{Link}\left(q^{j}, K\right)\right)\right)$. By the definition of $h_{q^{i}}^{\bullet}$ we have $S=\left(\mathrm{C} h_{q^{i}}^{\bullet} \times 1_{q^{i}}\right)^{-1}\left(\operatorname{Link}\left(q^{j}, K\right)\right)$. Also write $\bar{q}^{i}=\bar{q}^{l} \times \bar{q}^{j}$. Then $S \subset \mathbb{D}^{n-i} \times q^{l} \times\{x\}$. Identify $\bar{q}^{l}$ with $\bar{q}^{l} \times\{x\}$ and $x$ with a vertex of $\bar{q}^{l}$. By the definition of (geometric) link, we have that $S$ is the set of points $(a, b) \in \mathbb{D}^{n-i} \times q^{l}$ such that the segment $\left[x,\left(\mathrm{C} h_{q^{i}}(a), b\right)\right]$ has length $\epsilon$ (and is perpendicular to $q^{j}$ ) in $K$. Since $\mathrm{C} h_{q^{i}} \times 1_{q^{i}}$ is, by definition, radial in the first coordinate and the identity on the second, $S$ is the set of points $(a, b) \in \mathbb{D}^{n-i} \times q^{l}$ that satisfy the equation

$$
\epsilon^{2}=\delta^{2} d_{\mathbb{D}^{n-i}}^{2}(a, 0)+d_{\bar{q}^{l}}^{2}(b, x)
$$

where $d_{\mathbb{D}^{n-i}}$ and $d_{\bar{q}^{l}}$ are the euclidean distances on $\mathbb{D}^{n-i} \subset \mathbb{R}^{n-i}$ and $\bar{q}^{l} \subset \mathbb{R}^{l}$, respectively. Therefore $S$ is a submanifold of $\mathbb{D}^{n-i} \times q^{l}$. This proves the lemma. 
Now we prove the addendum. Recall that we are identifying a neighborhood of $q^{i}$ in $K$ (or $f\left(q^{i}\right)$ in $\left.M\right)$ with $\mathrm{C} \operatorname{Link}\left(q^{i}, K\right) \times q^{i}\left(\operatorname{respectively} f\left(\mathrm{C} \operatorname{Link}\left(q^{i}, K\right) \times q^{i}\right)\right)$.

Now we claim that the homothety map

$$
f\left(\left(\mathrm{C} \operatorname{Link}\left(q^{j}, K\right)-o\right) \times q^{j}\right) \times(0,1) \rightarrow f\left(\left(\mathrm{C} \operatorname{Link}\left(q^{j}, K\right)-o\right) \times q^{j}\right), \quad f(x, y, s) \mapsto f(s x, y)
$$

is smooth, where we are considering $f\left(\left(\mathrm{C} \operatorname{Link}\left(q^{j}, K\right)-o\right) \times q^{j}\right)$ with the structure $\mathcal{S}_{k}$. To see that the homothety above is smooth recall that we are writing $\bar{q}^{i}=\bar{q}^{l} \times \bar{q}^{j}$ and notice that the above map written in the chart $h_{q^{i}}^{\bullet}$ is $(a, b, c, s) \mapsto(s a, s b, c),(a, b, c, s) \in \mathbb{R}^{n-i} \times \mathbb{R}^{l} \times \mathbb{R}^{j} \times(0,1)$. (Here we are identifying $\bar{q}^{i}=\bar{q}^{l} \times \bar{q}^{j}$ with $[0,1]^{i}=[0,1]^{l} \times[0,1]^{j}$ so that $q^{j}$ corresponds to $\{0\} \times[0,1]^{j}$.) This shows that the homothety above smooth.

We also need the fact that the distance function $f(x, y) \mapsto|x|$, is smooth. Here $(x, y) \in$ $\left(\mathrm{C} \operatorname{Link}\left(q^{j}, K\right)-o\right) \times q^{j}$, and $|x|$ is the distance to the vertex in C Link $\left(q^{j}, K\right)$. To see this let $q^{i}$ so that $(x, y) \in q^{i}$. Using the notation in the previous paragraph we have the square of the distance function in the $h_{q^{i}}^{\bullet}$ chart is $(a, b, c) \mapsto \delta^{2} d_{\mathbb{D}^{n-i}}^{2}(a, 0)+d_{\bar{q}^{l}}^{2}(b, x)$ (as in equation (1)). This shows the square of the distance function (hence the distance function itself because it does not vanish) is smooth.

To prove the addendum just note that for $x \neq 0 \in \mathbb{D}^{k}$ we have

$$
h_{q^{j}}^{\bullet}(x, y)=f\left(|x| h_{q^{j}}\left(\frac{1}{|x|} x\right), y\right)
$$

where we are using the homothety and the distance function in the right side of the equality. Hence $h_{q^{j}}^{\bullet}$ is smooth (outside $q^{j}$ ). Again using charts it is straightforward to show that $h_{q^{j}}^{\bullet}$ is an immersion (outside $q^{j}$ ). Since $h_{q^{j}}$ is a (topological) embedding it follows that it is a smooth embedding. This proves the addendum.

\section{Remarks.}

1. Equation (1) in the all-right spherical case can be obtained using the spherical law of cosines. The equation is

$$
\epsilon=\cos \left(\delta d_{\mathbb{D}^{n-i}}(p, 0)\right) \cos \left(d_{\bar{q}^{l}}(q, x)\right) .
$$

2. Even if $f\left(\operatorname{Link}\left(q^{j}, K\right)\right)$ is diffeomorphic to an exotic sphere $\Sigma^{k-1}$ the Addendum to Lemma 1.2 remains true with one change: consider now $\mathbb{D}^{k}-0$ with the smooth structure given by the identification $\mathbb{D}^{k}-0=\Sigma^{k-1} \times(0,1)$.

Lemma 1.2 implies that the smooth structure $\mathcal{S}_{k}$ induces a smooth structure on the sphere $f\left(\operatorname{Link}\left(q^{j}, K\right)\right)$, but the smooth structure and the $P L$ structure (induced by $K$ ) are not necessarily compatible (see remark 7 in the introduction). If $k \leq 7, k \neq 5$ the $(k-1)$-sphere $f\left(\operatorname{Link}\left(q^{j}, K\right)\right)$, with the induced smooth structure, is diffeomorphic to the canonical sphere (see [8]). The next lemma says that the same is true for $k=5$.

Lemma 1.3. Let $j=n-5$ and consider the 4-sphere $f\left(\operatorname{Link}\left(q^{j}, K\right)\right)$ with the induced smooth structure from Lemma 1.2. Then $f\left(\operatorname{Link}\left(q^{j}, K\right)\right)$ is diffeomorphic to $\mathbb{S}^{4}$.

The proof of this lemma is given in the Appendix, but here is a quick sketch of the proof. Recall that $f\left(\operatorname{Link}\left(q^{n-5}, K\right)\right)$ with the $P L$ structure induced by $K$ is a $P L 4$-sphere (because $K$ induces a $P L$ triangulation on $M)$. But, as mentioned before, the $P L$ structure on $f\left(\operatorname{Link}\left(q^{n-5}, K\right)\right)$ 
induced by $\mathcal{S}_{5}$ may not coincide with the $P L$ structure induced by $K$. The trick here is to prove that these two $P L$ structures are $P L$ equivalent. The way to prove this is similar to the proof (given below, see condition $\mathbf{C}(k)$ ) that all structures $\mathcal{S}_{k}$ are diffeomorphic to the original $\mathcal{S}$.

We now prove the Main Theorem. Recall we are writing $j+k=n$. We begin with $k=1$ and define $\mathcal{S}_{1}=\left.\mathcal{S}\right|_{M-M_{n-1}}$. Then $\mathcal{A}_{1}=\left\{\left(h_{q^{n}}^{\bullet}, \mathbb{D}^{0} \times q^{n}\right)\right\}_{\bar{q}^{n} \in K}$ is a smooth atlas for $\mathcal{S}_{1}$. Using lemmas 1.2 and 1.3 (for the case $k=5$ ) we can construct $\mathcal{S}_{k}, k \leq 7$ inductively: $\mathcal{S}_{k+1}$ has an atlas $\mathcal{A}_{k+1}$ which is obtained from $\mathcal{A}_{k}$ by adding the charts $\left\{\left(h_{q^{j}}^{\bullet}, \mathbb{D}^{n-j} \times q^{j}\right)\right\}_{\bar{q}^{j} \in K}$, for some smoothings $h_{q^{j}}$ of the links of the $j$-cubes, such that $h_{q^{j}}: \mathbb{S}^{k-1} \rightarrow f\left(\operatorname{Link}\left(q^{j}, K\right)\right)$ is a diffeomorphism (which exists by 1.2 or 1.3$)$. Hence, by construction, we have that $\mathcal{A}_{k}=\left\{\left(h_{q^{i}}^{\bullet}, \mathbb{D}^{n-i} \times q^{i}\right)\right\}_{\bar{q}^{i} \in K, i>j}$, for $k \leq 7$.

Remark. As mentioned in the introduction every smooth manifold admits a smooth cubulation. Therefore Lemmas 1.2, 1.3 and the simple construction of the atlas $\mathcal{A}_{k}$ given above prove the following fact: the vanishing of $\Theta_{n}$ (the group of homotopy $n$-spheres), $n \leq 6, n \neq 4$, implies that every $P L n$-manifold is smoothable, for $n \leq 7$. Of course if the Smooth Poincare Conjecture in dimension 4 was true then we would not need Lemma 1.3.

The construction of the atlas $\mathcal{A}_{k}$ given above proves the Main Theorem for $n \leq 3$, since (diffeomorphism classes of) smooth structures are unique for $n \leq 3$. For $n=4$ it only remains to prove that the smooth structures $\mathcal{S}^{\prime}=\mathcal{S}_{5}$ and $\mathcal{S}$ are diffeomorphic, and this is done in the appendix (see A.2.3). Hence from now on we assume $n \geq 5$. We will prove by induction the following stronger statement. Let $\mathbf{S}^{\prime}(k), k \geq 4$, be the statement obtained from $\mathbf{S}(k)$ by adding the extra condition $\mathbf{C}(k)$ where:

$\mathbf{C}(4): \mathcal{S}_{4}$ extends to a smooth structure $\mathcal{S}_{4}^{\prime}$ on the whole $M$, and $\mathcal{S}_{4}^{\prime}$ is diffeomorphic to $\mathcal{S}$.

$\mathbf{C}(k>4): \quad \mathcal{S}_{k}$ extends to a smooth structure $\mathcal{S}_{k}^{\prime}$ on the whole $M$, and $\mathcal{S}_{k}^{\prime}$ is diffeomorphic to $\mathcal{S}_{k-1}^{\prime}$.

Recall that we have proved $\mathbf{S}(k)$ for $k \leq 7$. The next proposition shows our strategy to prove the Main Theorem.

Proposition 1.4. We have that

(1) Statement $\mathbf{C}(4)$ holds.

(2) Statements $\mathbf{C}(k)$ hold for $k=5,6$.

(3) Statement $\mathbf{S}^{\prime}(k-1)$ implies $\mathbf{S}(k)$.

(4) Statements $\mathbf{C}(k)$ and $\mathbf{S}(k+1)$ imply $\mathbf{C}(k+1)$ for $k \geq 6$.

Proof of Main Theorem assuming Proposition 1.4.

Just note that it follows from (2), (3), (4) and the fact that we have already proved $\mathbf{S}(7)$ that statement $\mathbf{S}^{\prime}(k)$ implies $\mathbf{S}^{\prime}(k+1)$, for $k \geq 6$. Thus induction shows that statement $\mathbf{S}^{\prime}(n+1)$ is true. This proves the Main Theorem assuming Proposition 1.4.

\section{Proof of Proposition 1.4.}


First we need a definition. A spindle neighborhood map of an open cube $q^{i}$ in $K$ is a topological embedding $\alpha: \mathbb{D}^{n-i} \times q^{i} \rightarrow K$ such that: (1) $\alpha(0, x)=x$ and (2) the diameters of the fibers $\alpha\left(\{x\} \times \mathbb{D}^{n-i}\right)$ (with respect to the path metric on $K$ ) tend uniformly to zero as $x$ tends to $\partial q^{i}$. (This terminology is essentially due to J. Munkres [9]). The image of $\alpha$ is a spindle neighborhood of $q^{i}$.

The proof of (1) uses some ingredients of the proof of Lemma 1.2, and it is given in the second part of the appendix.

We prove (2) next. Assume $\mathbf{C}(k-1)$ holds, where $k=5,6$. (Here we use that (1) holds.) Choose a spindle neighborhood for each $(j+1)$-cube $q^{j+1}$. We can assume all spindle neighborhoods to be disjoint. Since we are constructing $\mathcal{S}_{k}$ and $\mathcal{A}_{k}$ by induction, the smooth structure $\left.\mathcal{S}_{k}\right|_{M-M_{j+1}}$ coincides with $\mathcal{S}_{k-1}$. Hence the smooth structures $\mathcal{S}_{k}$ and $\mathcal{S}_{k-1}^{\prime}$ coincide on $M-M_{j+1}$. Let $q^{j+1}$ be a $(j+1)$-cube and pull back these two smooth structures to $\mathbb{D}^{k-1} \times q^{j+1}$ via the corresponding spindle map $\alpha_{q^{j+1}}$. Call these structures $\mathcal{B}$ and $\mathcal{C}$. They coincide outside $q^{j+1}=\{0\} \times q^{j+1}$.

Now, the Classification Theorem 10.1 of [7, p.194, says that concordance classes of smooth structures on $\mathbb{D}^{k-1} \times q^{j+1}$, modulo the complement $C$ of an open neighborhood of $q^{j+1}$ in $\mathbb{D}^{k-1} \times$ $q^{j+1}$, is in one-to-one correspondence with

$$
\left[\left(\mathbb{D}^{k-1} \times q^{j+1}, C\right), T O P / O\right]=\pi_{k-1}(T O P / O)
$$

From now on we chose $C$ to be a small spindle neighborhood of $q^{j+1}$ in $\mathbb{D}^{k-1} \times q^{j+1}$. Since $\pi_{k-1}(T O P / O)=\pi_{k-1}\left(K\left(\mathbb{Z}_{2}, 3\right)\right)=0$, for $4 \leq k-1 \leq 6([7 \mathrm{p} .201)$, we have that $\mathcal{B}$ and $\mathcal{C}$ are concordant modulo $C$. Therefore (see Theorem 4.1 on p.25 of [7]) $\mathcal{B}$ and $\mathcal{C}$ are isotopic modulo $C$. Consequently there is a diffeomorphism $\beta:\left(\mathbb{D}^{k-1} \times q^{j+1}, \mathcal{B}\right) \rightarrow\left(\mathbb{D}^{k-1} \times q^{j+1}, \mathcal{C}\right)$, which is the identity on $C$. Moreover $\beta$ is isotopic to the identity modulo $C$. Using these maps $\beta$ we can define a diffeomorphism $\left(M-M_{j}, \mathcal{S}_{k}\right) \rightarrow\left(M-M_{j}, \mathcal{S}_{k-1}^{\prime}\right)$ that extends to a homeomorphism $\phi: M \rightarrow M$ by declaring $\phi(x)=x$, for $x \in M_{j}$. Take now $\mathcal{S}_{k}^{\prime}$ to be the pull back of $\mathcal{S}_{k-1}^{\prime}$ by $\phi$. This proves (2).

To prove (3) we need the following result. Recall we are assuming $n \geq 5$.

Lemma 1.5. Assume $\mathbf{S}^{\prime}(k)$ holds. Consider the $(k-1)$-sphere $f\left(\operatorname{Link}\left(q^{j}, K\right)\right)$ with the induced smooth structure from Lemma 1.2. Then $f\left(\operatorname{Link}\left(q^{j}, K\right)\right)$ is diffeomorphic to $\mathbb{S}^{k-1}$.

Proof. Let $h_{q^{j}}: \mathbb{S}^{k-1} \rightarrow \operatorname{Link}\left(q^{j}, K\right)$ be a homeomorphism. Pull back the smooth structure $\mathcal{S}_{k}^{\prime}$ by the map $h_{q^{j}}^{\bullet}$ to obtain a smooth structure $\mathcal{B}$ on $\mathbb{D}^{n-j} \times q^{j}$. The Addendum to Lemma 1.2 (also see remark 2 before Lemma 1.3) implies that the smooth structure $\mathcal{B}$ is a product structure outside $q^{j}=\{0\} \times q^{j} \subset \mathbb{D}^{k} \times q^{j}$. By Lemma 1.3 we can assume $k-1>6$ and we can apply the Product Structure Theorem (see Theorem 5.1 on p.31 of [7]) to obtain a diffeomorphism $\left(\mathbb{D}^{k} \times q^{j}, \mathcal{C} \times \mathcal{S}_{\mathbb{R}^{j}}\right) \rightarrow\left(\mathbb{D}^{k} \times q^{j}, \mathcal{B}\right)$ for some smooth structure $\mathcal{C}$ on the disc $\mathbb{D}^{k}$. Here $\mathcal{S}_{\mathbb{R}^{j}}$ is the canonical smooth structure on $\mathbb{R}^{j}$. Furthermore, we can assume this diffeomorphism to be the identity outside a small neighborhood of $q^{j}$. Hence the smooth structure $\left.\mathcal{B}\right|_{\mathbb{S}^{k-1}}=\left.\mathcal{C}\right|_{\mathbb{S}^{k-1}}$ extends to a smooth structure on the whole disc. We can apply now the smooth $h$-cobordism Theorem to conclude that $\left.\mathcal{B}\right|_{\mathbb{S}^{k-1}}$ is diffeomorphic to the canonical structure on the sphere. This proves the lemma. 
We can prove (3) now: just use Lemma 1.5 and the addendum to Lemma 1.2 to construct $\mathcal{A}_{k}$ and $\mathcal{S}_{k}$ as done before. Therefore $\mathbf{S}^{\prime}(k-1)$ implies $\mathbf{S}(k)$.

It remains to prove (4), that is, we have to prove that $\mathbf{C}(k-1)$ and $\mathbf{S}(k) \operatorname{imply} \mathbf{C}(k)$, i.e. that $\mathcal{S}_{k}$ extends to the whole $M$. The proof is similar to the proof of (2), but first we may have to change the smoothings of the links of $j$-cubes (given in the proof of Lemma 1.5). The following lemma will indicate how to change the smoothing $h_{q^{j}}$. Recall that for $f: \mathbb{S}^{k} \rightarrow \mathbb{S}^{k}$ the map $\mathrm{C} f: \mathbb{D}^{k+1} \rightarrow \mathbb{D}^{k+1}$ is the cone of $f$ defined by $\mathrm{C} f(x)=|x| f\left(\frac{x}{|x|}\right), x \neq 0$ and $f(0)=0$.

Lemma 1.6. Let $\mathcal{D}$ be a smooth structure on the disc $\mathbb{D}^{k}, k \geq 6$. Assume $\mathcal{D}$ coincides with the canonical smooth structure outside a small neighborhood of the origin. Then there is a diffeomorphism $g: \mathbb{S}^{k-1} \rightarrow \mathbb{S}^{k-1}$ such that the smooth structure $(\mathrm{C} g)^{*} \mathcal{D}$ is diffeomorphic to the canonical one, by a diffeomorphism that is the identity outside a small neighborhood of the origin.

Proof. Let $G: \mathbb{D}^{k} \rightarrow\left(\mathbb{D}^{k}, \mathcal{D}\right)$ be a diffeomorphism. Since, by hypothesis, $\mathcal{D}$ coincides with the canonical smooth structure outside a small neighborhood of the origin, we can assume that $G$ is radial outside a small neighborhood of the origin (to see this use the uniqueness of collars or deformation of vector fields techniques). Take $g=\left.G\right|_{\mathbb{S}^{k-1}}$ and note that $\left((\mathrm{C} g)^{-1} \circ G\right)^{*}(\mathrm{C} g)^{*} \mathcal{D}=$ $G^{*} \mathcal{D}$ is the canonical smooth structure on $\mathbb{D}^{k}$. Moreover $(\mathrm{C} g)^{-1} \circ G$ is the identity outside a small neighborhood of the origin. This proves Lemma 1.6.

We now prove (4). Assume $\mathbf{C}(k)$ and $\mathbf{S}(k+1)$ hold. Let $\mathcal{B}=\mathcal{B}\left(h_{q^{j}}\right)$ be the pull-back smooth structure $\left(h_{q^{j}}^{\bullet}\right)^{*} \mathcal{S}_{k}^{\prime}$. On the other hand note that, by construction, the pull-back smooth structure $\left(h_{q^{j}}^{\bullet}\right)^{*} \mathcal{S}_{k+1}$ is the canonical smooth structure on $\mathbb{D}^{k} \times q^{j}$. Since $\mathcal{S}_{k}^{\prime}$ extends $\mathcal{S}_{k}$, and $\mathcal{S}_{k}$ and $\mathcal{S}_{k+1}$ coincide on $M-M_{j}$, we have that $\mathcal{B}$ is a smooth structure on $\mathbb{D}^{k} \times q^{j}$ that coincides with the canonical one outside $q^{j}$.

For a self-homeomorphism $g$ on $\mathbb{S}^{k-1}$ write $g^{\bullet}=(\mathrm{C} g) \times 1_{q^{j}}$, which is a self-homeomorphism on $\mathbb{D}^{k-1} \times q^{j}$.

Claim. There is a self-homeomorphism $g$ on $\mathbb{S}^{k-1}$ such that $\mathcal{B}\left(h_{q^{j}} \circ g\right)=\left(g^{\bullet}\right)^{*} \mathcal{B}$ is diffeomorphic to the canonical smooth structure on $\mathbb{D}^{k} \times q^{j}$, via a diffeomorphism that is the identity outside a small spindle neighborhood of $q^{j}$ in $\mathbb{D}^{k} \times q^{j}$.

Proof of claim. Recall that we are denoting by $\mathcal{S}_{\mathbb{R}^{j}}$ the canonical smooth structure on $\mathbb{R}^{j}$. Using the Product Structure Theorem (see Theorem 5.1 in [7, p.31), we get a diffeomorphism

$$
\gamma:\left(\mathbb{D}^{k} \times q^{j}, \mathcal{D} \times \mathcal{S}_{\mathbb{R}^{j}}\right) \rightarrow\left(\mathbb{D}^{k} \times q^{j}, \mathcal{B}\right)
$$

for some smooth structure $\mathcal{D}$ on the disc $\mathbb{D}^{k}$. Furthermore, $\gamma$ is the identity outside a small spindle neighborhood of $q^{j}$ and isotopic to the identity (by an isotopy that is constant outside a small spindle neighborhood of $q^{j}$ ). Apply Lemma 1.6 to $\mathcal{D}$ to obtain a diffeomorphism $g$. Note that $\left(g^{\bullet}\right)^{-1} \circ \gamma \circ g^{\bullet}$ is a diffeomorphism between the smooth structures $\left(\gamma \circ g^{\bullet}\right)^{*} \mathcal{B}$ and $\left(g^{\bullet}\right)^{*} \mathcal{B}$, and this diffeomorphism is the identity on the complement of a small neighborhood of $q^{j}$. But by Lemma 1.6 we have that $\left(\gamma \circ g^{\bullet}\right)^{*} \mathcal{B}=\left(g^{\bullet}\right)^{*}\left(\mathcal{D} \times \mathcal{S}_{\mathbb{R}^{j}}\right)=(\mathrm{C} g)^{*} \mathcal{D} \times \mathcal{S}_{\mathbb{R}^{j}}$ which is diffeomorphic to the canonical smooth structure on $\mathbb{D}^{k} \times q^{j}$, via a diffeomorphism that is the identity on the complement of a small neighborhood of $q^{j}$. Therefore we have proved that $\left(g^{\bullet}\right)^{*} \mathcal{B}$ is diffeomorphic to the canonical smooth structure on $\mathbb{D}^{k} \times q^{j}$, via a diffeomorphism that is the identity outside a small neighborhood of $q^{j}$ in $\mathbb{D}^{k} \times q^{j}$ (not necessarily spindle). Call this diffeomorphism $\eta$, that 
is, $\eta^{*}\left(g^{\bullet}\right)^{*} \mathcal{B}$ is diffeomorphic to the canonical smooth structure, and $\eta$ is the identity outside a small neighborhood of $q^{j}$. We have to change $\eta$ by one that is the identity outside a small spindle neighborhood of $q^{j}$.

To be specific let $\eta$ be the identity outside $B \times q^{j}$, where $B \subset \mathbb{D}^{k}$ is an open ball centered at 0 of small radius. Let $\theta$ be a self diffeomorphism of $\mathbb{D}^{k} \times q^{j}$ and $V$ a small spindle neighborhood of $q^{j}$ such that: (1) $\theta$ is of the form $\theta(u, v)=\left(\theta_{v}(u), v\right)$, that is, $\theta$ maps the fibers $\mathbb{D}^{k} \times\{v\}$ to themselves, (2) $V=\coprod_{v \in q^{j}} B_{v} \times\{v\}$, where $B_{v} \subset \mathbb{D}^{k}$ is an open ball centered at 0 of small radius $r_{v}$, and $r_{v} \rightarrow 0$ uniformly as $v \rightarrow \partial q^{j}$, (3) $\theta$ is the identity inside a very small spindle neighborhood $U$ of $q^{j}$, with $\bar{U} \subset V$, (4) $B \subset \theta_{v}\left(B_{v} \times\{v\}\right)$. By (3) and the fact that $\mathcal{B}$ coincides with the canonical structure outside $q^{j}$ we have that $\theta$ is also a self diffeomorphism of $\left(\mathbb{D}^{k} \times q^{j}, \mathcal{B}\right)$. It is now straightforward to verify that $\theta^{-1} \circ \eta \circ \theta$ is a diffeomorphism between $\left(g^{\bullet}\right)^{*} \mathcal{B}$ and the canonical smooth structure on $\mathbb{D}^{k} \times q^{j}$, and that this map is the identity outside the small spindle neighborhood $V$ of $q^{j}$. This proves the claim.

From the claim it follows that if we replace the link smoothing $h_{q^{j}}$ by the new link smoothing $h_{q^{j}} \circ g$ and take $\mathcal{B}=\mathcal{B}\left(h_{q^{j}} \circ g\right)$ instead of $\mathcal{B}=\mathcal{B}\left(h_{q^{j}}\right)$ we get that the new structure $\mathcal{B}$ is now diffeomorphic to the canonical one modulo the complement of a small spindle neighborhood of $q^{j}$. That is there is a diffeomorphism $\beta=\beta_{q^{j}}: \mathbb{D}^{k} \times q^{j} \rightarrow\left(\mathbb{D}^{k} \times q^{j}, \mathcal{B}\right)$ that is the identity outside a small spindle neighborhood of $q^{j}$. We can now proceed as in the the proof of (2) (i.e. the case $k \leq 7)$ and use the maps $\beta_{q^{j}}$ to obtain a diffeomorphism $\left(M-M_{j-1}, \mathcal{S}_{k+1}\right) \rightarrow\left(M-M_{j-1}, \mathcal{S}_{k}^{\prime}\right)$ that extends to a homeomorphism $\phi: M \rightarrow M$ by declaring $\phi(x)=x$, for $x \in M_{j}$. Take now $\mathcal{S}_{k+1}^{\prime}$ to be the pull-back of $\mathcal{S}_{k}^{\prime}$ by $\phi$. This proves (4) and completes the proof of Proposition 1.4

\section{Manifolds with codimension zero singularities.}

In this section we treat the case of manifolds with a one point singularity. The case of manifolds with many (isolated) point singularities is similar.

Let $Q$ be a smooth manifold with a one point singularity $q$, that is, $Q-\{q\}$ is a smooth manifold and there is a topological embedding $\mathrm{C}_{1} N \rightarrow Q$, with $o_{\mathrm{C} N} \mapsto q$, which is a smooth embedding outside the vertex $o_{\mathrm{C} N}$. Here $N=\left(N, \mathcal{S}_{N}\right)$ is a closed smooth manifold (with smooth structure $\mathcal{S}_{N}$ ). Also $\mathrm{C}_{1} N$ is the closed cone of $N \times[0,1] / N \times\{0\}$. We write $\mathrm{C}_{1} N \subset Q$ and sometimes we shall identify $\mathrm{C}_{1} N-\left\{o_{\mathrm{C} N}\right\}$ with $N \times(0,1]$. We say that the singularity $q$ of $Q$ is modeled on $\mathrm{C} N$. Also we say that $(K, f)$ is a smooth cubulation of $Q$ if

(i) $K$ is a cubical complex.

(ii) $f: K \rightarrow Q$ is a homeomorphism. Write $f(p)=q$ and $L=\operatorname{Link}(p, K)$.

(iii) $\left.f\right|_{\sigma}$ is a smooth embedding for every cube $\sigma$ not containing $p$.

(iv) $\left.f\right|_{\sigma-\{p\}}$ is a smooth embedding for every cube $\sigma$ containing $p$.

(v) $L$ is $P L$ homeomorphic to $\left(N, \mathcal{S}_{N}\right)$.

Many of the definitions and results given in previous sections still hold (with minor changes) in the case of manifolds with a one point singularity: 
(1) A link smoothing for $L=\operatorname{Link}(p, K)$ (or $p$ ) is just a homeomorphism $h_{p}: N \rightarrow L$. A set of link smoothings for $K$ is a set of link smoothings for the sphere links plus a link smoothing for $L$.

(2) Given a set of link smoothings for $K$ we get a set of normal charts as before. For the vertex $p$ we mean the cone map $h_{p}^{\bullet}=f \circ \mathrm{C} h_{p}: \mathrm{C} N \rightarrow Q$. We will also denote the restriction of $h_{p}^{\bullet}$ to $\mathrm{C} N-\left\{o_{\mathrm{C} N}\right\}$ by the same notation $h_{p}^{\bullet}$. As before $\left\{h_{\sigma}^{\bullet}\right\}_{\sigma \in K}$ is a (topological) normal atlas on $Q$ with respect to $K$. The atlas on $Q$ is smooth if all transition functions are smooth, where for the case $h_{p}^{\bullet}: \mathrm{CN}-\left\{o_{\mathrm{C} N}\right\} \rightarrow Q-\{q\}$ we are identifying $\mathrm{C} N-\left\{o_{\mathrm{C} N}\right\}$ with $N \times(0,1]$ with the product smooth structure obtained from some smooth structure $\tilde{\mathcal{S}}_{N}$ on $N$. A smooth normal atlas on $Q$ with respect to $K$ induces, by restriction, a smooth normal structure on $Q-\{q\}$ with respect to $K-\{p\}$ (this makes sense even though $K-\{p\}$ is not, strictly speaking, a cube complex).

(3) We say that the set $\left\{h_{\sigma}\right\}$ is smooth if the atlas $\mathcal{A}=\left\{h_{\sigma}^{\bullet}\right\}_{\sigma \in K}$ is smooth. In this case we say that the smooth atlas $\mathcal{A}$ (or the induced smooth structure, or the set $\left\{h_{\sigma}\right\}$ ) is correct with respect to $N$ if $\mathcal{S}_{N}$ and $\tilde{\mathcal{S}}_{N}$ are diffeomorphic.

The Main Theorem also holds in this context:

Theorem 4.1. Let $Q$ be a smooth manifold with one point singularity $q$ modeled on $\mathrm{C} N$, where $N$ is a closed smooth manifold. Let $(K, f)$ be a smooth cubulation of $Q$. Then $Q$ admits a normal smooth structure with respect to $K$, which restricted to $Q-\{q\}$ is diffeomorphic to $Q-\{q\}$. Moreover this normal smooth structure is correct with respect to $N$ if

(a) $\operatorname{dim} N \leq 4$.

(b) $\operatorname{dim} N>4$ and the Whitehead group $W h(N)$ of $N$ vanishes.

Proof. The proof of the existence part is the same as the proof of the Main Theorem. Moreover it also follows from the proof of the Main Theorem that the smooth structures $\mathcal{S}, \mathcal{S}^{\prime}$ on $Q-\{q\}$ are diffeomorphic. Here $\mathcal{S}$ is the given smooth structure on $Q-\{q\}$ and $\mathcal{S}^{\prime}$ is the (restriction of) normal smooth structure on $Q-\{q\}$ with respect to $K$.

It remains to prove that the normal smooth structures are correct with respect to $N$. This is certainly true if $n \leq 3$ (because of the uniqueness of differentiable structures, up to diffeomorphisms, in dimensions $\leq 3$ ). We assume $n \geq 4$.

From the proof of the Main Theorem we see that $S=f(\operatorname{Link}(p, K))$ is a smooth submanifold of $\left(Q-\{q\}, \mathcal{S}^{\prime}\right)$ (see Lemma 1.2). Let $\mathcal{S}_{S}^{\prime}$ be the smooth structure on $S$ induced by $\mathcal{S}^{\prime}$. Note that the link smoothing $h_{p}:\left(N, \tilde{\mathcal{S}}_{N}\right) \rightarrow\left(S, \mathcal{S}_{S}^{\prime}\right)$ is a diffeomorphism (see (2) above). Note also that $\mathcal{S}_{S}^{\prime}$ is a normal smooth structure on $S$ with respect to $L$, as can be verified from the proof of the Main Theorem applied to $S$ and $L$ (actually the link smoothings are the same; for more details see 1.3.5 in [11]). If $n=4$ then Applying A.2.1 we get that $\left(S, \mathcal{S}_{S}^{\prime}\right)$ is $P L$ homeomorphic to $L$. On the other hand condition (v) above imply that $\left(N, \mathcal{S}_{N}\right)$ is also $P L$ homeomorphic to $L$. It follows that all three $\left(S, \mathcal{S}_{S}^{\prime}\right),\left(N, \tilde{\mathcal{S}}_{N}\right),\left(N, \mathcal{S}_{N}\right)$ are $P L$ equivalent. Since we are in dimension four (see [2], 6]) all three spaces above are diffeomorphic and (a) follows. 
We prove (b). By hypothesis there is an embedding $\left(N, \mathcal{S}_{N}\right) \rightarrow(Q-\{q\}, \mathcal{S})$ whose image lies near $q$. Since $\mathcal{S}$ is diffeomorphic to $\mathcal{S}^{\prime}$, we get that there is an embedding $\left(N, \mathcal{S}_{N}\right) \rightarrow\left(Q-\{q\}, \mathcal{S}^{\prime}\right)$ whose image lies near $q$. But near $q$ the smooth structure $\mathcal{S}^{\prime}$ is diffeomorphic to the product $I_{\epsilon} \times S$ with smooth structure $\mathcal{S}_{I_{\epsilon}} \times \mathcal{S}_{N}^{\prime}$, where $I_{\epsilon}=(0, \epsilon)$. And this product is diffeomorphic to $I_{\epsilon} \times N$ with smooth structure $\mathcal{S}_{I_{\epsilon}} \times \tilde{\mathcal{S}}_{N}$. Therefore there is an embedding $\left(N, \mathcal{S}_{N}\right) \rightarrow\left(I_{\epsilon} \times N, \mathcal{S}_{I_{\epsilon}} \times \tilde{\mathcal{S}}_{N}\right)$. Hence, by a standard topological argument, $\left(N, \tilde{\mathcal{S}}_{N}\right)$ and $\left(N, \mathcal{S}_{N}\right)$ are smoothly h-cobordant. Consequently the proof of (b) is obtained by applying the h-cobordism Theorem. This proves the Theorem.

\section{Appendix. Proof of Lemma 1.3 and 1.4 (1).}

In the first part of this appendix we prove Lemma 1.3, and in the final part we prove statement (1) of Proposition 1.4 (this is condition $\mathbf{C}(4)$ ). Recall that we are using the following definition: a non-degenerate $P D$ map $K \rightarrow M, K$ a complex and $M$ a smooth manifold, is such that its restriction to each simplex (or cube) is smooth and its derivative at every point is injective ([10] p. 75).

Here are some ideas of the proof of Lemma 1.3. Recall that $f\left(\operatorname{Link}\left(q^{n-5}, K\right)\right)$ with the $P L$ structure induced by $K$ is a $P L 4$-sphere (because $K$ induces a $P L$ triangulation on $M$ ). Also recall that the $P L$ structure on $f\left(\operatorname{Link}\left(q^{n-5}, K\right)\right)$ induced by $\mathcal{S}_{5}$ may not coincide with the $P L$ structure induced by $K$. To prove Lemma 1.3 we will prove that these two $P L$ structures are $P L$ equivalent. The way to prove this is similar to the proof of condition $\mathbf{C}(k)$ that says that all structures $\mathcal{S}_{k}$ are diffeomorphic to the original $\mathcal{S}$. The problem is that these diffeomorphisms do not respect the links, hence the cannot be restricted to give diffeomorphisms (hence $P L$ homeomorphisms) of links. But we will show that these diffeomorphisms do preserve a "cube" version of the links. We introduce this concept in the next subsection.

\section{A.1. Cubic Links.}

Let $\epsilon>0$ and write

$$
\begin{array}{ll}
I^{k}(\epsilon)=[0, \epsilon]^{k} \subset \mathbb{R}^{k} & S^{k}(\epsilon)=\mathbb{S}^{k-1}(\epsilon) \cap I^{k}(\epsilon) \\
C^{k}(\epsilon)=\mathbb{D}^{k}(\epsilon) \cap I^{k}(\epsilon) & F^{k}(\epsilon)=\left\{\left(x_{1}, \ldots x_{k+1}\right) \in I^{k}(\epsilon), x_{i}=1 \text { for some } i\right\}
\end{array}
$$

where $\mathbb{S}^{k-1}(\epsilon)$ and $\mathbb{D}^{k}(\epsilon)$ are the $(k-1)$-sphere and the $k$-disc of radius $\epsilon$ respectively. Then $S^{k}(\epsilon)$ is a canonical all-right spherical simplex of dimension $k$ and radius $\epsilon$ and $I^{k}(\epsilon)$ is the canonical $k$-cube of side-length $\epsilon$. Note that for $\epsilon \leq \epsilon^{\prime}<<\epsilon^{\prime \prime}$ we have $C^{k}(\epsilon) \subset I^{k}\left(\epsilon^{\prime}\right) \subset C^{k}\left(\epsilon^{\prime \prime}\right)$. In this situation by deforming along rays emanating from the origin we can construct a radial $P D$ selfhomeomorphism $\Theta=\Theta\left(\epsilon, \epsilon^{\prime}, \epsilon^{\prime \prime}\right)$ on $C^{k}\left(\epsilon^{\prime \prime}\right)$ such that (1) it is the identity on $C^{k}(\epsilon / 2)$, and near $S^{k}\left(\epsilon^{\prime \prime}\right) \quad(\mathbf{2})$ sends $I^{k}\left(\epsilon^{\prime}\right)$ to $C^{k}(\epsilon)$. The inverse of $\Theta$ is also $P D$.

Let $K^{k}$ be a cube complex, $\sigma^{i} \in K$ and $x \in \dot{\sigma}^{i}$. Write $j=k-i$. Recall that the $\epsilon$-link $\operatorname{Link}_{\epsilon}\left(\dot{\sigma}^{i}, K\right)$ and $\epsilon$-star $\mathrm{C} \operatorname{Link}_{\epsilon}(\dot{\sigma}, K)$ of $\sigma^{i}$ at $x$ are built by gluing copies of $S^{j}(\epsilon)$ and $C^{j}(\epsilon)$ respectively. The $\epsilon$-cubic link $\square \operatorname{Link}_{\epsilon}(\dot{\sigma}, K)$ of $\sigma^{i}$ at $x$ is obtained by gluing copies of $F^{j}(\epsilon)$ in the same way as the $S^{j}(\epsilon)$. Analogously, the $\epsilon$-cubic cone link $\square \mathrm{CL}^{2} \operatorname{Link}_{\epsilon}(\dot{\sigma}, K)$ of $\sigma^{i}$ at $x$ is obtained by gluing copies of $I^{j}(\epsilon)$ in the same way as the $C^{j}(\epsilon)$. Then we can write

$$
\mathrm{CLink}_{\epsilon}(\dot{\sigma}, K) \subset \square \mathrm{CLink}_{\epsilon^{\prime}}(\dot{\sigma}, K) \subset \mathrm{CLink}_{\epsilon^{\prime \prime}}(\dot{\sigma}, K)
$$


for $\epsilon \leq \epsilon^{\prime}<<\epsilon^{\prime \prime}$. By gluing the maps $\Theta$ mentioned above simplexwise we obtain a radial PD selfhomeomorphism $\Theta$ (we use the same letter) on $\mathrm{C} \operatorname{Link}_{\epsilon^{\prime \prime}}(\dot{\sigma}, K)$ with similar properties: (1) it is the identity on $\mathrm{C} \operatorname{Link}_{\epsilon / 2}(\dot{\sigma}, K)$, and near $\mathrm{C} \operatorname{Link}_{\epsilon^{\prime \prime}}(\dot{\sigma}, K)$ (2) $\operatorname{sends}_{\square} \mathrm{C} \operatorname{Link}_{\epsilon^{\prime}}(\dot{\sigma}, K)$ to $\mathrm{C} \operatorname{Link}_{\epsilon}(\dot{\sigma}, K)$. Again the inverse of $\Theta$ is also $P D$.

A.1.1. The spherical case. Consider the upper-half space $\left\{x_{n+1}>0\right\} \subset \mathbb{R}^{n+1}$. For each $i=1, \ldots, n$ define the smooth function $\theta_{i}:\left\{x_{n+1}>0\right\} \rightarrow(-\pi / 2, \pi / 2)$ by $\theta_{i}\left(x_{1}, \ldots, x_{n+1}\right)=$ $\tan ^{-1}\left(\frac{x_{i}}{x_{n+1}}\right)$. Note that $\theta_{i}$ is the composition of the projection $\left(x_{1}, \ldots, x_{n+1}\right) \mapsto\left(x_{n+1}, x_{i}\right)$, with angle function of polar coordinates. Write $\theta=\left(\theta_{1}, \ldots, \theta_{n}\right)$. We denote the restriction of $\theta$ to the upper hemisphere $\mathbb{S}_{+}^{k}=\mathbb{S}^{k} \cap\left\{x_{n+1}>0\right\}$ by the same letter $\theta$. Let $p=e_{n+1} \in \mathbb{S}_{+}^{k}$ (the north pole). Then $\theta(p)=0 \in \mathbb{R}^{k}$. Note that the derivative $D \theta_{p}$ is the identity matrix. (To see this let $\alpha_{1}(s)=(\sin (s), 0, \ldots, 0, \cos (s))$. Then $\theta_{1}(\alpha(s))=s$, hence $D \theta_{p} \cdot e_{1}=\left(\theta \circ \alpha_{1}\right)^{\prime}(0)=e_{1}$. Similarly for $i>1$.) It follows that $\theta$ is a diffeomorphism near $p$ and it has an inverse $\vartheta: \mathbb{B}_{\mu}\left(\mathbb{R}^{k}\right) \rightarrow \mathbb{S}^{k}$, where $\mathbb{B}_{\mu}\left(\mathbb{R}^{k}\right)$ is the ball of (small) radius $\mu$ in $\mathbb{R}^{k}$ centered at the origin.

If $K$ is an all-right-spherical complex instead of a cube one, similar concepts can be defined as above, with the $\epsilon$ objects replaced by their images in $\mathbb{S}^{k}$ by $\vartheta$, were we take $\epsilon$ small enough.

A.2. Proof of Lemma 1.3. We write $L=\operatorname{Link}\left(q^{j}, K\right), j=n-5$ and $N=f(L)$. Then $L$ is an all-right spherical complex $P L$ homeomorphic to the 4 -sphere $\mathbb{S}^{4}$. From the proof of the Main Theorem it can be checked that the smooth structure $\mathcal{S}_{N}^{\prime}$ on $N=f(L)$ given by Lemma 1.2 has an atlas of the form

$$
\mathcal{A}=\left\{\left(h_{\sigma^{i}}^{\bullet}, \mathbb{D}^{4-i} \times \dot{\sigma}^{i}\right)\right\}_{\sigma^{i} \in L}
$$

That is, $\mathcal{A}$ is a normal smooth atlas, hence $\mathcal{S}_{N}^{\prime}$ is a normal smooth structure. Since $L$ is $P L$ homeomorphic to $\mathcal{S}^{4}$, Lemma 1.3 is implied by the following result and the fact that $P L$ homeomorphic smooth 4-manifolds are diffeomorphic ([2], [6]).

Proposition A.2.1. Let $\mathcal{S}^{\prime}$ is a normal smooth structure on the manifold $N^{4}$ with respect to $L$, where $L$ is a cubulation or all-right spherical triangulation of $N$. Then $L$ is $P L$ homeomorphic to $\left(N^{4}, \mathcal{S}^{\prime}\right)$.

We will give a proof of the proposition for the case of $L$ being a cube complex. The proof for the all-right spherical case is obtained replacing cubic links by the objects in A.1.1.

Proof of A.2.1. Since in what follows the map $f$ is not essential, from now on we identify $L$ and $N$ via $f$ and write $L=N$. The identity map $L \rightarrow N$ is not $P D$ (see remark 7 after the Main Theorem). To prove that $\left(N, \mathcal{S}_{N}^{\prime}\right)$ is $P L$ homeomorphic to $L$ we will modify the identity $L \rightarrow N$ to a non-degenerate $P D$ homeomorphism $\phi: L \rightarrow\left(N, \mathcal{S}_{N}^{\prime}\right)$, which implies that $\phi: L^{\prime} \rightarrow\left(N, \mathcal{S}_{N}^{\prime}\right)$ is a smooth triangulation of $\left(N, \mathcal{S}_{N}^{\prime}\right)$. Here $L^{\prime}$ is a subdivision of $L$. We will need the following Lemma.

Lemma A.2.2. Let $\varphi: J \rightarrow \mathbb{S}^{k}$ be a smooth triangulation of $\mathbb{S}^{k}$ and $\psi: \mathbb{S}^{k} \rightarrow \mathbb{S}^{k}$ homeomorphism which is non-degenerate $P D$ with respect to $J$. Then $\varphi$ extends to a smooth triangulation $\varphi^{\prime}$ : $J^{\prime} \rightarrow \mathbb{D}^{k+1}$ and $\psi$ extends to a homeomorphism $\psi^{\prime}: \mathbb{D}^{k+1} \rightarrow \mathbb{D}^{k+1}$ which is non-degenerate PD with respect to $J^{\prime}$.

Addendum to Lemma A.2.2. We can assume that $J^{\prime}$ and $\psi^{\prime}$ are radial outside a small ball 
centered at the origin.

Remark. Simply coning $\varphi$ does not work because differentiability would be lost at the origin. The idea of the proof is to first extend $\varphi$ radially (but not to the origin). Then, near the origin approximate this extension by a simplicial map, and this simplicial map can be extended to the origin using coning.

Proof. Take $K=J \times[\eta, 1]$ and $f(x, t)=t \varphi(x)$, which is a non-degenerate $P D$ homeomorphism onto its image (hence a smooth triangulation of its image [10], p.77). Here $\eta>0$ is small. Let $\epsilon$ sufficiently small so that any $\epsilon$-approximation of $f$ is also a $P D$ embedding ([10], Th. 8.8). Take $K_{1}=J \times[\eta, 2 \eta]$ and $g: K_{1}^{\prime} \rightarrow \mathbb{R}^{k+1}$ the secant $\delta$-approximation of $\left.f\right|_{K_{1}^{\prime}}\left([10]\right.$, p. 87) where $K_{1}^{\prime}$ is a subdivision of $K_{1}$ so that $g$ is also an embedding ([10, Th. 8.8). Taking $\delta$ even smaller if necessary and using 9.8 of [10] (with $\epsilon$ as above) we get a subdivision $K^{\prime}$ of $J \times[\eta, 1]$ and a non-degenerate $P D$ homeomorphism $h$ on $K^{\prime}$ which coincides with $f$ outside (say) $J \times[0,3 \eta]$ and is simplicial on $K_{1}^{\prime}$ (which is a subdivision of $J \times[\eta, 2 \eta]$ ). Let $J^{\prime \prime}$ be the subdivision of $J \times\{\eta\} \subset K$ induced by $K^{\prime}$. Take $J^{\prime}=\left(\mathrm{C} J^{\prime \prime}\right) \coprod_{J^{\prime \prime}} K^{\prime}$ and extend $h$ simplicially on $\mathrm{C} J^{\prime \prime} \subset J^{\prime}$ to obtain $h^{\prime}: J^{\prime} \rightarrow \mathbb{D}^{k+1}$. Then $h^{\prime}$ is a non-degenerate $P D$ map and if $\epsilon$ is sufficiently small $h^{\prime}$ is also a homeomorphism. It can be checked that $J^{\prime}$ depends only on the bounds of the derivatives of $\varphi$. Therefore we can assume that when we apply the same argument to $\psi \circ \varphi: J \rightarrow \mathbb{S}^{k}$ we obtain a similar map $h^{\prime \prime}$ defined also on the same $J^{\prime}$, that is $h^{\prime \prime}: J^{\prime} \rightarrow \mathbb{D}^{k+1}$ is non-degenerate, is radial outside a small neighborhood of the cone point of $J^{\prime}$ and coincides with $\psi \circ \varphi$ on $J \subset J^{\prime}$. Finally take $\psi^{\prime}=h^{\prime \prime} \circ\left(h^{\prime}\right)^{-1}$. This proves Lemma A.2.2.

Note that, by construction, the complex $J^{\prime}$ and the map $\psi^{\prime}$ are radial outside a ball centered at the origin; this proves the addendum to Lemma A.2.2.

We now continue the proof of Proposition A.2.1. To simplify we write $N=\left(N, \mathcal{S}_{N}^{\prime}\right)$. Now, the identity map $L \rightarrow N$ is already $P D$ outside the two skeleton $L^{2}$ of $L$ (it is $P D$ on the interior of the 3-simplices). For $\sigma \in L$ and $\delta>0$ denote by $\sigma_{\delta}$ the set of points in $\sigma$ that lie at a distance $>\delta$ from $\partial \sigma$. Hence $\sigma_{\delta} \subset \dot{\sigma}$.

Using the charts $\left(h_{\sigma^{i}}^{\bullet}, \mathbb{D}^{4-i} \times \dot{\sigma}\right)$ we identify $\mathbb{D}^{4-i} \times \dot{\sigma}$ with its image by $h_{\sigma^{i}}^{\bullet}$. In particular, for $x \in \dot{\sigma}$, we are identifying $\mathbb{D}^{n-i} \times\{x\}$ with the $\mathrm{C} \operatorname{Link}(\sigma, K)=\mathrm{CLink}_{\epsilon}(\dot{\sigma}, K)$ based at $x$, for some $\epsilon>0$.

We choose $\delta>0$ small and $\epsilon>0$ even smaller to make sure that the open sets $\mathbb{D}^{2} \times \sigma_{\delta}$, for all 2-simplices $\sigma^{2} \in L$, have disjoint closures. Let $\sigma^{2} \in L$ be a 2-simplex in $L$ and $x \in \sigma_{\delta}^{2}$. Now, the identity $\mathbb{D}^{2} \times\{x\}=\mathrm{C} \operatorname{Link}\left(\dot{\sigma}^{2}, L\right) \rightarrow \mathbb{D}^{2} \times\{x\}$ is not, in general, a $P D$ map at the origin $(0, x) \in \mathbb{D}^{2} \times\{x\}$ (in fact it looks like the spherical version of the example given in the introduction; see also remark 7 in the introduction). But using Lemma A.2.2 we can modify the identity $\mathbb{D}^{2} \times\{x\}=\mathrm{C} \operatorname{Link}\left(\dot{\sigma}^{2}, L\right) \rightarrow \mathbb{D}^{2} \times\{x\}$ near the origin $(0, x) \in \mathbb{D}^{2} \times\{x\}$ to make it a non-degenerate $P D$ homeomorphism. Crossing with the identity $1_{\dot{\sigma}}$ we obtain a non-degenerate $P D$ self-homeomorphism of $\mathbb{D}^{2} \times \sigma_{\delta}^{2}$, which is the identity outside a small neighborhood of $\sigma_{\delta}^{2}$. Patching all these maps for all $\sigma^{2} \in L$ together with the identity we obtain a non-degenerate $P D$ homeomorphism $\psi$ on $L(2)=\left(L-L^{2}\right) \cup\left(\bigcup_{\sigma^{2} \in L} \mathbb{D}^{2} \times \sigma_{\delta}^{2}\right)$. Note that $\psi$ is still a product map on each $\mathbb{D}^{2} \times \sigma_{\delta}^{2}$. 
Now let $\sigma^{1} \in L$ be a 1 -simplex and $x \in \sigma_{\delta^{\prime}}^{1}$, where $\delta^{\prime}$ is small but with $\delta<<\delta^{\prime}$ (we may have to take $\delta$ and $\epsilon$ even smaller). Also let $\epsilon^{\prime}$ be such that $\epsilon<<\epsilon^{\prime}$ so that the $\epsilon^{\prime}$-link at $x$ is such that $\operatorname{Link}_{\epsilon^{\prime}}\left(\dot{\sigma}^{1}, L\right) \subset L(2)$, hence we can apply $\psi$ to $\operatorname{Link}_{\epsilon^{\prime}}\left(\dot{\sigma}^{1}, L\right)$. We would like to use Lemma A.2.2 to extend $\psi$ to a $P D$ map on $\mathrm{C} \operatorname{Link}_{\epsilon^{\prime}}\left(\dot{\sigma}^{1}, L\right)$ (thus also near $\sigma_{\delta}^{1}$ ) but the problem is that $\psi$ does not map $\operatorname{Link}_{\epsilon^{\prime}}\left(\dot{\sigma}^{1}, L\right)$ to itself. To correct this we work with cubic links. Note that $\square$ Link $_{\epsilon^{\prime}}\left(\dot{\sigma}^{1}, L\right) \subset L(2)$. And, since $\psi$ is still a product map on each $\mathbb{D}^{2} \times \sigma_{\delta}^{2}$ we have that now $\psi$ maps $\square$ Link $_{\epsilon^{\prime}}\left(\dot{\sigma}^{1}, L\right)$ to itself. We can now apply Lemma A.2.2 to the restriction of $\Theta \psi \Theta^{-1}$ to Link $_{\epsilon^{\prime}}\left(\dot{\sigma}^{1}, L\right)$ to obtain a non-degenerate $P D$ extension $\psi^{\prime}$ defined on $\mathrm{C}$ Link $_{\epsilon^{\prime}}\left(\dot{\sigma}^{1}, L\right)$. Take now $\Theta^{-1} \psi^{\prime} \Theta$ and cross this map with $1_{\sigma_{\delta^{\prime}}^{1}}$ to extend $\psi$ near $\sigma_{\delta^{\prime}}^{1}$. Doing this for all $\sigma^{1} \in L$ and patching these maps with the previous $\psi$ we obtain a non-degenerate $P D$ homeomorphism $\psi$ on $L(1)=L(2) \cup\left(\bigcup_{\sigma^{1} \in L} \mathbb{D}^{3} \times \sigma_{\delta}^{1}\right)$. Note that $\psi$ is still a product map on each $\mathbb{D}^{2} \times \sigma_{\delta^{\prime \prime}}^{2}$ and on each $\mathbb{D}^{3} \times \sigma_{\delta^{\prime}}^{1}$ (where $\delta^{\prime \prime}$ is slightly smaller than $\delta$ ). Therefore $\psi$ maps $\square \operatorname{Link}_{\epsilon^{\prime \prime \prime}}\left(\dot{\sigma}^{0}, L\right)$ to itself, for a suitable choice of $\epsilon^{\prime \prime \prime}$. To extend $\psi$ to the whole of $L$ we proceed one step further (now for 0 -simplices $\sigma^{0}$ ) in a similar way in the case for 1-simplices $\sigma^{1}$. This proves Proposition A.2.1 and Lemma 1.3.

The argument used in the proof above gives the following corollaries. In the first corollary we use the notation in the Main Theorem and its proof.

Corollary A.2.3. Let $f: K \rightarrow\left(M^{4}, \mathcal{S}\right)$ be a PD non-degenerate homeomorphism. Let $\mathcal{S}^{\prime}=\mathcal{S}_{5}$ (as in the proof of the Main Theorem). Then $(M, \mathcal{S})$ and $\left(M, \mathcal{S}^{\prime}\right)$ are diffeomorphic.

Proof. We have that $\mathcal{S}^{\prime}$ is a normal smooth structure on $M$, with respect to $K$. By Proposition A.2.1 $\left(M, \mathcal{S}^{\prime}\right)$ is $P L$ homeomorphic to $K$. On the other hand, since $K$ is a smooth cubulation (or all-right spherical triangulation) of $(M, \mathcal{S})$, we have that $K$ is $P L$ homeomorphic to $(M, \mathcal{S})$. Therefore $(M, \mathcal{S})$ and $\left(M, \mathcal{S}^{\prime}\right)$ are $P L$ homeomorphic, and, since we are in dimension 4 ([2], 6]), it follows that $(M, \mathcal{S})$ and $\left(M, \mathcal{S}^{\prime}\right)$ are diffeomorphic. This proves the corollary.

We can also generalize the arguments in the proof of Lemma 1.3 to higher dimensions. Suppose $K$ is a (cube of all-right spherical) complex of dimension $n$. We can choose the $\epsilon$ 's and $\delta$ 's above properly and define $K(n-2)=\left(K-K_{n-2}\right) \cup\left(\cup_{\sigma^{n-2} \in K} \mathbb{D}^{2} \times \sigma_{\delta}^{n-2}\right)$ and $K(k)=K(k+1) \cup$ $\left(\bigcup_{\sigma^{j} \in L} \mathbb{D}^{n-j} \times \sigma_{\delta}^{j}\right)$. From the definitions we have the equalities

$$
\begin{aligned}
& K-K(n-2)=K_{n-2}-\bigcup_{\sigma^{n-2} \in K} \sigma_{\delta}^{n-2} \supset K_{n-3} \\
& K-K(k)=(K-K(k+1))-\bigcup_{\sigma^{k} \in K} \sigma_{\delta}^{k} \supset K_{k-1}
\end{aligned}
$$

where the inclusion on top holds because the $\sigma_{\delta}^{n-2}$ are disjoint from $K_{n-3}$, and the inclusion on the second row follows from the first and induction. Note that these inclusions are homotopy equivalences. Hence we get

$$
K(k+1) \subset K-K_{k}
$$

Moreover, it is straightforward to verify that this inclusion is a homotopy equivalence. 
Corollary A.2.5. Let $f: K \rightarrow\left(M^{n}, \mathcal{S}\right)$ be a PD non-degenerate homeomorphism. Let $\mathcal{S}_{k}$ be as in the proof of of the Main Theorem satisfying condition $\mathbf{S}(k)$. Then there is a non-degenerate $P D$ homeomorphism $\psi: K(k+1) \rightarrow\left(f(K(k+1)), \mathcal{S}_{k}\right)$. In particular we have that (take $\left.k=-1\right)$ $(K, \psi)$ is a smooth cubulation of $\left(M, \mathcal{S}^{\prime}\right)$, for some $\psi$.

Proof. Take $K=L$ in the proof of Lemma 1.3 above, but now assume $K$ of arbitrary dimension. Using exactly the same arguments (i.e. use Lemma A.2.2 and cubic links) given there we can extend the induction to all values of $k$. This proves the corollary.

We now prove statement (1) of Proposition 1.4.

Proof of $\mathbf{C}(4)$. The smooth structure $\mathcal{S}_{4}$ is defined on $M-M_{n-4}$ and we have to prove that it extends to a smooth structure $\mathcal{S}_{4}^{\prime}$ on $M$, and $\mathcal{S}_{4}^{\prime}$ is diffeomorphic to $\mathcal{S}$.

Write $A=M-M_{n-4}$. Let $L(j)$ be as in corollary A.2.5 and write $B=L(n-3) \subset K$, $D=f(B) \subset M$. Note that $D \subset A$. Since the inclusion in A.2.4 is a homotopy equivalence we have:

(A.2.6) the inclusion $D \hookrightarrow A$ is a homotopy equivalence.

By corollary A.2.5 there is a non-degenerate $P D$ homeomorphism $\psi: B \rightarrow\left(D,\left.\mathcal{S}_{4}\right|_{D}\right)$. We have that $\psi$ extends to a homeomorphism (we use the same letter) $\psi: K \rightarrow M$.

Remark. The last statement follows from the inductive proof of Corollary A.2.5, taking $j=-1$. That is, the $\psi$ constructed for $K(j)$ extends the $\psi$ constructed in the previous step for $K(j+1)$, but on a slightly smaller smaller $K(j)$; see the proof of Lemma 1.3. But we can also construct this extension using Alexander's trick (beyond codimension 3) instead of Lemma A.2.2.

Write $f^{\prime}=\left.f\right|_{B}$ and note that, by hypothesis, $f^{\prime}: B \rightarrow(D, \mathcal{S})$ is also a non-degenerate $P D$ homeomorphism. Consider the smooth structures $\mathcal{S}_{\psi}=\psi^{*} \mathcal{S}_{4}$ and $\mathcal{S}_{f}=\left(f^{\prime}\right)^{*} \mathcal{S}=\left.\left(f^{*} \mathcal{S}\right)\right|_{B}$ on $B$. The $P L$ structure on $B$ (induced by $K$ ) is thus Whitehead compatible with both smooth structures $\mathcal{S}_{\psi}$ and $\mathcal{S}_{f}$. But $A=M-M_{n-4}$ has the homotopy type of a 3 -complex hence, by A.2.5, so do $D$ and $B$. Since $P L / O$ is 6 -connected it follows from the theory of smoothings of $P L$-manifolds (see Theorem 4.2 in the second essay in [6]) that $\mathcal{S}_{\psi}$ and $\mathcal{S}_{f}$ are concordant. Consequently, pushing forward everything to $D$ by $\psi$ we have that the smooth structures $\left.\mathcal{S}_{4}\right|_{D}$ and $\mathcal{S}^{\prime \prime}=\psi_{*} \mathcal{S}_{f}$ are concordant. Note that $\mathcal{S}^{\prime \prime}=\left(\left(f \circ \psi^{-1}\right)^{*} \mathcal{S}\right)_{D}$ hence we get that

\section{(A.2.7) the smooth structures $\left.\mathcal{S}_{4}\right|_{D}$ and $\left(\left(f \circ \psi^{-1}\right)^{*} \mathcal{S}\right)_{D}$ on $D$ are concordant}

It follows from A.2.6, A.2.7 and the theory of smoothings of topological manifolds (see the Classification Theorem 10.1, p .194, in [7] and its naturality for restrictions) that the smooth structures $\mathcal{S}_{4}$ and $\left(\left(f \circ \psi^{-1}\right)^{*} \mathcal{S}\right)_{A}$ on $A$ are concordant. Therefore we can find a self-homeomorphism $g$ on $A$ such that $\left(\left(f \circ \psi^{-1}\right)^{*} \mathcal{S}\right)_{A}=g^{*} \mathcal{S}_{4}$. Moreover we can assume (see Theorem 4.1, p. 25, in [7]) that that $d_{M}(x, g(x)) \rightarrow 0$, as $x \rightarrow \partial A$ (here $d_{M}$ is any metric on $M$ inducing the topology on $M)$. Therefore we can extend $g$ to a self-homeomorphism on $M$ by defining $g(x)=x$, for $x \notin A$. 
To finish the proof just take $\mathcal{S}_{4}^{\prime}=\left(f \circ \psi^{-1} \circ g^{-1}\right)^{*} \mathcal{S}$, which is defined on the whole $M$ and extends $\mathcal{S}_{4}$. This proves statement $\mathbf{C}(4)$.

\section{References}

[1] M. Bridson and A. Haeflinger, Metric spaces of non-positive curvature, Springer-Verlag (1999).

[2] J. Cerf, Sur les difféomorphismes de la sphère de dimension trois $\left(\Gamma_{4}=0\right)$. Lecture Notes in Mathematics 53. Springer verlag, Berlin-New York, 1968.

[3] R. M. Charney and M. W. Davis, Strict hyperbolization, Topology 34 (1995), 329-350.

[4] M. W. Davis and T. Januszkiewicz, Hyperbolization of polyhedra, J. Differential Geom. 34 (1991), 347-388.

[5] F. T. Farrell and W.C. Hsiang, The whitehead group of poly-(finite or cyclic) groups, J. London math. Soc. (2) 24 (1982), 308-324

[6] M. W. Hirsch and B. Mazur, Smoothings of piecewise linear manifolds. Annals of Math. Studies 80. Princeton University Press (1974).

[7] R.C. Kirby and L.C. Siebenmann, Foundational Essays on Topological Manifolds, Smoothings, and Triangulations, Annals of Math. Studies no.88, Princeton University Press, Princeton (1977).

[8] M. Kervaire and J. Milnor, Groups of homotopy spheres I, Annals of Math 77 (1963), 504-537.

[9] J. R. Munkres, Obstructions to the smoothing of piecewise-differentiable homeomorphisms, Annals of Mathematics 72 (1960), 521-554.

[10] J. R. Munkres, Elementary Differential Topology. Annals of Math. Studies 54, Princeton University Press (1963).

[11] P. Ontaneda, Normal smoothings for Charney-Davis strict hyperbolizations. Arxiv: 1406.1737.

[12] P. Ontaneda, Riemannian hyperbolization. Arxiv: 1406.1730.

Pedro Ontaneda

SUNY, Binghamton, N.Y., 13902, U.S.A. 